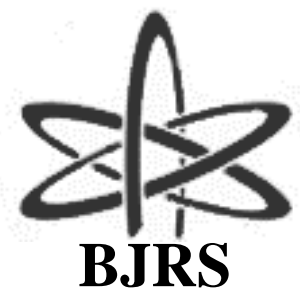

BRAZILIAN JOURNAL

$\mathrm{OF}$

RADIATION SCIENCES

08-03 (2020) 01-18

\title{
Solução das equações da cinética pontual de nêutrons via Método de Fator de Integração Implícito com fonte
}

\section{externa}

\author{
Zandonáa J. C., Petersen ${ }^{a}$ C. Z., Tumelero ${ }^{a}$ F., Schaun ${ }^{a}$ N., Schramm ${ }^{a}$ M. \\ ${ }^{a}$ Universidade Federal de Pelotas, Campus Capão do Leão, Programa de Pós-Graduação em Modelagem Matemática \\ Caixa Postal 354 - CEP 96001-970, Capão do Leão - RS, Brasil, Prédio 05 - $3^{\circ}$ andar - Sala 302. \\ jaime.zandona@ufpel.edu.br
}

\begin{abstract}
RESUMO
Os autores apresentam uma abordagem semi-analítica para obter soluções para as equações da cinética pontual de nêutrons. A reatividade dependente do tempo é aproximada por aproximação constante por partes em tempo discretizado, e o sistema de equações diferenciais é resolvido por um fator de integração. O termo de fonte externa na solução possui uma integral, que é resolvida numericamente pela interpolação de polinômios de Lagrange e uma fórmula simples de quadratura. Mesmo que a fonte externa apresente não linearidade nas equações, a metodologia é apropriada. A metodologia proposta é utilizada em casos de referência padrão e seus resultados são comparados com os da literatura.

Palavras-Chave: Cinética pontual de nêutrons, Fator integrante, Aproximação constante por partes, Fonte externa.
\end{abstract}

\begin{abstract}
The authors present a semi-analytical approach to obtain solutions to the neutron point kinetics equations. The time dependent reactivity is approximated by piecewise constant approximation in discretized time, and the system of differential equations is solved by an integrating factor. The external source term in the solution has an integral, which is numerically solved by Lagrange polynomials interpolation and a simple quadrature formula. Even if the external source presents nonlinearity in the equations, the methodology is appropriate. The proposed methodology is used in benchmark cases and its results are compared with the ones in the literature.
\end{abstract}

Keywords: Neutron point kinetics, Integrating factor, Piecewise constant approximation, External source. 


\section{INTRODUCÃO}

A energia nuclear tem um espaço importante na matriz energética mundial devido à crescente demanda por energia e as inúmeras vantagens proporcionadas. Dentre as mais relevantes, vale destacar a sua aplicação na área da medicina e indústria com a produção de radioisótopos por reatores de pesquisa. Além disso, os reatores nucleares de potência, que geram energia elétrica, utilizam pouco material para produzir grandes quantidades de energia e atendem aos objetivos do Protocolo de Kyoto e, mais recentemente, o Acordo de Paris [1], por não emitir dióxido de carbono (CO2), provocando pouco ou quase nenhum impacto sobre a biosfera. Entretanto, a sustentabilidade ainda é o maior desafio no desenvolvimento de tecnologias nucleares inovadoras como opção em longo prazo.

A utilização de regeneração e ciclos fechados para assegurar o suprimento de combustível para geração de eletricidade com energia nuclear tem sido o principal alvo no desenvolvimento de reatores rápidos e permanece como um importante objetivo a ser atingido.

Para tornar a energia nuclear uma fonte sustentável de energia, com relação aos recursos naturais e rejeitos nucleares deve ser considerada a utilização de ciclos fechados de Plutônio em reatores rápidos, bem como a transmutação/incineração de actinídeos menores (AM) e produtos de fissão de longa vida (PFLV) em vários tipos de reatores nucleares, tais como os Accelerator driven system (ADS). Nesse contexto surgiu o conceito de ADS, que são sistemas dedicados à transmutação de resíduos de alta atividade para reduzi-los dos repositórios geológicos [2-5]. Uma razão importante para o interesse na utilização destes sistemas é a possibilidade de geração de energia da fissão e, ao mesmo tempo, incinerar rejeitos radioativos. Além disso, outras vantagens são: ausência de acidentes de reatividade (trabalham num sistema subcrítico), alta capacidade de transmutação, baixa produção de rejeitos e melhor utilização dos recursos naturais em longo prazo. Os sistemas acionados por fonte consistem num sistema rápido subcrítico mantido em estado estacionário por uma fonte externa de nêutrons.

A Equação de Transporte de Boltzmann dependente do tempo que leva em conta além dos nêutrons prontos também os nêutrons atrasados [6,7] define de forma íntegra a população de nêutrons, mas possui o obstáculo de ser uma equação de difícil resolução. Uma aproximação usada é a Equação da Difusão de Nêutrons (EDN) que é adequada para cálculos globais em física de reatores quando ocorrem mudanças espaciais e temporais na distribuição de nêutrons. 
Nos últimos anos, vários algoritmos foram desenvolvidos para lidar, tanto com a Equação da Difusão, como com a Equação de Transporte de Nêutrons. Observa-se também que os tempos de simulação foram continuamente reduzidos graças ao desenvolvimento de recursos computacionais. No entanto, para situações mais realistas, a solução da Equação de Transporte e Difusão de Nêutrons considerando meio heterogêneo, problema multidimensional, multigrupo de energia, coeficientes dependentes do tempo e inclusão de precursores de nêutrons atrasados, ainda é um desafio do ponto de vista computacional e uma aproximação é uma alternativa. Isso se torna mais evidente no caso de simulações que levam em consideração a termohidráulica do problema, em que o cálculo se torna cada vez mais custoso, devido ao feedback de temperatura, que torna o problema não linear. Em alguns casos de interesse prático (grandes reatores de potência), a evolução da potência de um reator nuclear pode ser suficientemente bem aproximada usando a Equação da Cinética Pontual de Nêutrons (ECPN).

O modelo da Cinética Pontual de Nêutrons ainda desempenha um papel muito importante em física de reatores, à medida que pode ser usado, quando resolvido adequadamente, para uma previsão quase em tempo real do transiente de potência do reator. Isso permite controle e intervenção oportunos na usina, a fim de evitar a ocorrência de acidentes graves. Como é sabido, o problema clássico da ECPN é a rigidez decorrente da diferença entre as ordens de grandeza dos tempos de geração dos nêutrons prontos e atrasados após uma fissão, o que resulta na exigência de incrementos muito pequenos no intervalo de tempo para soluções numéricas às equações cinéticas.

Assumindo total separabilidade do tempo e espaço é possível chegar às ECPN, que é um sistema de equações diferenciais ordinárias (EDO) acopladas que descrevem a densidade de nêutrons e concentração de precursores de nêutrons atrasados.

Certamente, se as equações têm coeficientes constantes, soluções analíticas exatas são facilmente estabelecidas, mas são pouco efetivas quando os coeficientes variam com o tempo. Tem havido muitas pesquisas focadas em eliminar o problema da rigidez ao longo dos anos e vários métodos foram especialmente adaptados para resolver o problema de valor inicial para sistemas rígidos de equações diferenciais ordinárias.

Como ilustração, podemos citar os que seguem: Chao \& Attard desenvolveram o Método do Confinamento da Rigidez que desacopla a rigidez das equações diferenciais para precursores e confina na equação da densidade de nêutrons, que pode ser resolvida analiticamente [3]; Sánchez 
utiliza o Método de Runge-Kutta Generalizado de quarta ordem [8]; Aboanber \& Nahla utilizam aproximações de Padé para encontrar a função exponencial das equações e as raízes da fórmula de Inhour são usadas como autovalores da matriz da cinética pontual [2]; Aboanber \& Nahla apresenta uma solução em séries de potências, obtendo-se a solução recursivamente [1]; o algoritmo CORE de Quintero-Leyva utiliza transformada de Laplace e Teorema de expansão de Heaviside com as raízes da equação de Inhour [9]; em Nahla apresenta-se o Método Exponencial Analítico Generalizado que baseia-se nos autovalores e autovetores da matriz dos coeficientes [10]; no Método Numérico Integral de Li et al., utilizando a Melhor Função Base (BBF), é feita a integração exata nas equações para a densidade de nêutrons em cada passo de tempo com uma formulação totalmente implícita [11]; Nahla apresenta o método New Analytic Method (NAM), baseado nas raízes da Equação de Inhour, usando o Método da Eliminação Gaussiana para resolver as Equações da Cinética Pontual de Nêutrons [12]; Método da Decomposição de Petersen et al., é um método analítico que separa a matriz dos coeficientes em duas, uma com a dependência temporal e a outra com as constantes, transformando as equações em um conjunto de problemas recursivos semelhantes as equações com reatividade constante [13]; em Ganapol [5], Ganapol\&Picca [6] e Ganapol [7], apresenta-se a Técnica de Alta Precisão baseada na aproximação constante por partes feita por Kinard \& Allen [14]. A alta precisão é alcançada pela introdução de uma submalha para a avaliação numérica das integrais, corrigindo o termo fonte; Picca et al., desenvolvem a Aproximação Constante por Partes Melhorado (EPCA) com a vantagem que possibilita, através de um ciclo iterativo, a correção do pressuposto básico do método PCA [15]; no método de Silva et al., aplica-se o procedimento semelhante à Petersen [13], mas a solução pode ser determinada sem a dependência de tempos anteriores [16]; Tumelero et al., aplica o Método da Aproximação Polinomial (PAM) que consiste em expandir as variáveis em séries de potências, considerando a reatividade como uma função constante em um intervalo de tempo relativamente pequeno em torno de um ponto ordinário, na qual no primeiro intervalo aplicam-se as condições iniciais do problema e utiliza-se a continuação analítica para determinar as soluções dos próximos passos [17].

Com o referencial apresentado percebe-se que muitos métodos já foram implementados para resolver a ECPN, entretanto o acoplamento de fontes de spallation em modelos ADS com núcleos subcríticos ainda é um assunto pouco explorado e precisa ser melhor entendido e estudado. Com o passar do tempo, novos modelos, métodos e parâmetros nucleares são propostos para estimar a 
população de nêutrons dentro do núcleo de um reator nuclear de potência, por isso deve-se sempre realizar testes para verificar, tanto a capacidade do modelo, quanto a do método a ser empregado, a fim de prever situações específicas, definindo limites de validade (qualificação do modelo e método). Esses testes são realizados utilizando-se problemas de referência (padrões) em física de reatores chamados de problemas benchmark. Mais recentemente uma atenção especial tem sido dada aos ADS [18-19].

Portanto, neste trabalho resolve-se as ECPN, considerando 6 grupos de precursores de nêutrons atrasados, reatividade constante e dependente do tempo com um termo fonte definido de forma genérica, podendo depender até da própria densidade de nêutrons.

\section{METODOLOGIA}

Partindo das ECPN, considerando seis grupos de nêutrons atrasados com fonte externa tem-se:

$$
\begin{aligned}
\frac{d n(t)}{d t} & =\frac{\rho(t)-\beta}{\Lambda} n(t)+\sum_{i=1}^{6} \lambda_{i} C_{i}(t)+S(n(t)) \\
\frac{d C_{i}(t)}{d t} & =\frac{\beta_{i}}{\Lambda} n(t)-\lambda_{i} C_{i}(t),
\end{aligned}
$$

com as seguintes condições iniciais

$$
\begin{aligned}
n(0) & =n_{0} \\
C_{i}(0) & =\frac{\beta_{i}}{\lambda_{i} \Lambda} n_{0},
\end{aligned}
$$

para $i=1: 6$, onde, $n(t)$ dado em $\left[\mathrm{cm}^{-3}\right]$ é a densidade de nêutrons no tempo $t, C_{i}(t)$ dado em $\left[\mathrm{cm}^{-3}\right]$ é a concentração de precursores de nêutrons atrasados para o grupo $i$ de precursores no tempo $t, \rho(t)$ é a reatividade no instante $t, \beta$ é a fração de nêutrons atrasados, $\beta_{i}$ são as frações de nêutrons atrasados para o grupo $i$ de precursores, $\lambda_{i}$ dado em $\left[s^{-1}\right]$ são as constantes de decaimento para o 
grupo $i$ de precursores, $\Lambda$ dado em $[s]$ é o tempo médio de geração entre o nascimento dos nêutrons e posterior absorção e $S(n(t))$ dado em $\left[\mathrm{cm}^{-3}\right]$ é o termo fonte, podendo representar uma fonte externa de nêutrons genérica.

Assim, reescrevendo a equação (1) na forma matricial, tem-se:

$$
\frac{d \mathbf{y}(t)}{d t}=\boldsymbol{A}(t) \mathbf{y}(t)+\mathbf{S}(\mathbf{y}(t))
$$

onde

$$
\mathbf{y}(t)=\left(\begin{array}{c}
n(t) \\
C_{1}(t) \\
\vdots \\
C_{6}(t)
\end{array}\right), \quad \boldsymbol{A}(t)=\left(\begin{array}{cccc}
\frac{\rho(t)-\beta}{\Lambda} & \lambda_{1} & \cdots & \lambda_{6} \\
\frac{\beta_{1}}{\Lambda} & -\lambda_{1} & 0 \cdots & 0 \\
\vdots & 0 & & \vdots \\
\frac{\beta_{6}}{\Lambda} & 0 & 0 & -\lambda_{6}
\end{array}\right) \quad \boldsymbol{e} \quad \boldsymbol{S}(\mathbf{y}(t))=\left(\begin{array}{c}
S_{0}(n(t)) \\
0 \\
\vdots \\
0
\end{array}\right)
$$

Para resolver a equação (3) utiliza-se a ideia do método Fator de Integração Implícito (FII)[5]. Esse método é obtido a partir da multiplicação de um fator de integração apropriado, a fim de manter a parte não homogênea em uma forma implícita, na qual é aproximado por um método de interpolação.

Para evitar trabalhar com o fator de integração $e^{-\int A(t) d t}$ na equação (3), utiliza-se a ideia do piecewise constant approximation (PCA) presente em [15]. Ou seja, considera-se a reatividade, único termo dependente do tempo da matriz $\mathbf{A}(t)$, como uma função constante por partes. Em outras palavras, discretiza-se o tempo em $n+1$ pontos da forma $t_{0}<t_{1}<t_{2}<\cdots<t_{s}$ e aproxima-se a reatividade por $\rho(t)=\rho\left(t_{n+1}\right)$, na qual $n=(0: s-1)$ para cada subintervalo. Aqui $n$ é o número de pontos discretizados no domínio que depende do tamanho do espaçamento da malha $(\Delta t)$ e do ponto 
final do intervalo a ser escolhido $t_{s}$. Com isso, utiliza-se a solução no ponto $t_{n}$ como condição inicial para o próximo, fazendo uso da continuação analítica.

Assim, para cada subintervalo do domínio, tem-se uma matriz constante associada a cada ponto final dos subintervalos, ou seja, para cada subintervalo tem-se a reatividade constante. Logo, tem-se o fator integrante $e^{-A t}$ de forma genérica para todos os subintervalos. Multiplicando a equação (3) por este fator integrante, tem-se:

$$
e^{-A t} \frac{d \mathbf{y}(t)}{d t}=e^{-A t} \boldsymbol{A} \mathbf{y}(t)+e^{-A t} \mathbf{S}(\mathbf{y}(t))
$$

Reescrevendo a equação (5) e utilizando a regra do produto, tem-se:

$$
\frac{d\left(e^{-A t} \mathbf{y}(t)\right)}{d t}=e^{-A t} \mathbf{S}(\mathbf{y}(t))
$$

Integrando entre $t_{n}$ e $t_{n+1}$, sendo $t_{n+1}=t_{n}+\Delta t$, em ambos os lados desta equação, obtém-se:

$$
\int_{t_{n}}^{t_{n+1}} \frac{d\left(e^{-A t} \mathbf{y}(t)\right)}{d t} d t=\int_{t_{n}}^{t_{n+1}} e^{-A t} \mathbf{S}(\mathbf{y}(t)) d t
$$

Fazendo uma mudança de variável, $\tau=t-t_{n}$, tem-se:

$$
\left.e^{-A t} \mathbf{y}(t)\right|_{t_{n}} ^{t_{n+1}}=\int_{0}^{\Delta t} e^{-A t} \mathbf{S}\left(\mathbf{y}\left(t_{n}+\tau\right)\right) d \tau
$$

Aplicando os limites de integração em (8), fica-se com:

$$
e^{-A\left(t_{n}+\Delta t\right)} \mathbf{y}\left(t_{n}+\Delta t\right)-e^{-A t_{n}} \mathbf{y}\left(t_{n}\right)=e^{-A t_{n}} \int_{0}^{\Delta t} e^{-A \tau} \mathbf{S}\left(\mathbf{y}\left(t_{n}+\tau\right)\right) d \tau
$$


Multiplicando a equação (9) por $e^{A\left(t_{n}+\Delta t\right)}$, tem-se:

$$
\mathbf{y}\left(t_{n}+\Delta t\right)-e^{A \Delta t} \mathbf{y}\left(t_{n}\right)=e^{A \Delta t} \int_{0}^{\Delta t} e^{-A \tau} \mathbf{S}\left(\mathbf{y}\left(t_{n}+\tau\right)\right) d \tau
$$

Assim:

$$
\mathbf{y}_{n+1}=e^{A \Delta t} \mathbf{y}_{n}+e^{A \Delta t} \int_{0}^{\Delta t} e^{-A \tau} \mathbf{S}\left(\mathbf{y}\left(t_{n}+\tau\right) d \tau,\right.
$$

onde $\mathbf{y}\left(t_{n}\right)=\mathbf{y}_{n}$ é utilizado, usualmente, para aproximação de $\mathbf{y}\left(t_{n}\right)$. Aqui cabe ressaltar que $\mathbf{y}\left(t_{0}\right)$ é a condição inicial do problema e os demais $\mathbf{y}_{n}$ são soluções conhecidas do passo anterior. Quer tratarse a parte não homogênea de forma implícita construindo um esquema que se baseia em aproximar $e^{-\boldsymbol{A} \tau} \mathbf{S}\left(\mathbf{y}\left(t_{n}+\tau\right)\right)$ através de uma interpolação polinomial que envolva $t_{n+1}$, por isso define-se:

$$
G(\tau)=e^{-A \tau} \mathbf{S}\left(\mathbf{y}\left(t_{n}+\tau\right)\right)
$$

Para construir um esquema de integração de ordem $r$ de erro de truncamento, aproxima-se $G(\tau)$ por um polinômio de Lagrange de ordem $(r-1)$, com pontos de interpolação $t_{n+1}, t_{n}, \ldots, t_{n+2-r}$.
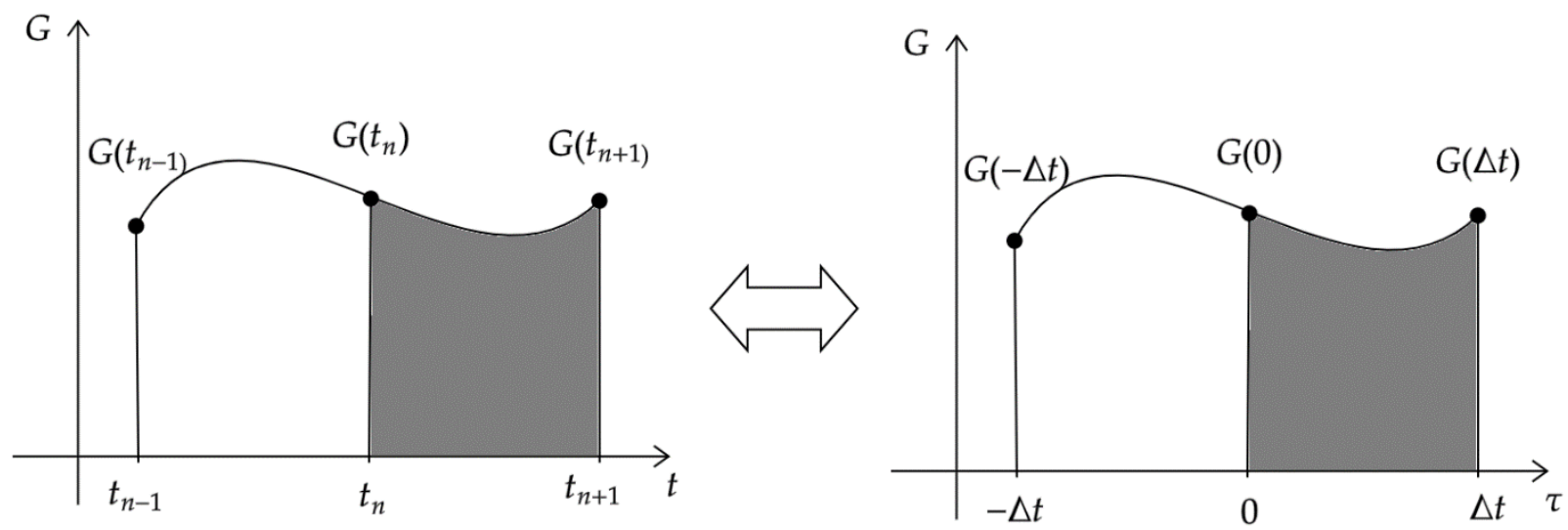

Figura1: Ilustração genérica de uma interpolação por Lagrange considerando 3 pontos 
$\mathrm{Na}$ mudança de variáveis trabalha-se com os pontos de interpolação $\Delta t, 0,-\Delta t, \ldots,(2-r) \Delta t$ : então:

$$
P(\tau)=\sum_{i=1}^{r} L_{r, i}(\tau) G((2-i) \Delta t)=\sum_{i=-1}^{r-2} L_{r, i}(\tau) G((-i) \Delta t)
$$

onde $L_{r, i}$ são os polinômios de Lagrange, dados por:

$$
L_{r, i}(\tau)=\prod_{\substack{j=-1 \\ j \neq i}}^{r-2} \frac{\tau-(-j \Delta t)}{(-i \Delta t-(-j \Delta t)}=\prod_{\substack{j=-1 \\ j \neq i}}^{r-2} \frac{\tau+j \Delta t}{(j-i) \Delta t} .
$$

Considerando em particular $r=3$ tem-se que:

$$
P(\tau)=\sum_{i=-1}^{1} L_{3, i}(\tau) G((-i) \Delta t)=L_{3,-1}(\tau) G(\Delta t)+L_{3,0}(\tau) G(0)+L_{3,1}(\tau) G(-\Delta t)
$$

onde

$$
\left\{\begin{array}{l}
G(\Delta t)=e^{-\boldsymbol{A} \Delta t} \mathbf{S}\left(\mathbf{y}\left(\Delta t+t_{n}\right)\right)=e^{-\boldsymbol{A} \Delta t} \mathbf{S}\left(\mathbf{y}_{n+1}\right) \\
G(0)=\mathbf{S}\left(\mathbf{y}\left(t_{n}\right)\right)=\mathbf{S}\left(\mathbf{y}_{n}\right) \\
G(-\Delta t)=e^{A \Delta t} \mathbf{S}\left(\mathbf{y}\left(t_{n}-\Delta t\right)\right)=e^{A \Delta t} \mathbf{S}\left(\mathbf{y}\left(t_{n-1}\right)\right)=e^{A \Delta t} \mathbf{S}\left(\mathbf{y}_{n-1}\right)
\end{array}\right\}
$$

Assim, para o esquema de ordem $r$, tem-se:

$$
P(\tau)=\sum_{i=-1}^{r-2} e^{i A \Delta t} \mathbf{S}\left(\mathbf{y}_{n-i}\right) \prod_{\substack{j=-1 \\ j \neq i}}^{r-2} \frac{\tau+j \Delta t}{(j-i) \Delta t} \quad ; 0 \leq \tau \leq \Delta t
$$

Portanto, da equação (11), fica-se com: 


$$
\mathbf{y}_{n+1}=e^{A \Delta t} \mathbf{y}_{n}+e^{A \Delta t} \int_{0}^{\Delta t} \sum_{i=-1}^{r-2} e^{i \Delta \Delta t} \mathbf{S}\left(\mathbf{y}_{n-i}\right) \prod_{\substack{j=-1 \\ j \neq i}}^{r-2} \frac{\tau+j \Delta t}{(j-i) \Delta t} d \tau \quad ; 0 \leq \tau \leq \Delta t
$$

Desta forma, obtém-se o esquema do método FII geral como:

$$
\mathbf{y}_{n+1}=e^{A \Delta t} \mathbf{y}_{n}+\Delta t\left(\alpha_{n+1} \mathbf{S}\left(\mathbf{y}_{n+1}\right)+\sum_{i=0}^{r-2} \alpha_{n-i} \mathbf{S}\left(\mathbf{y}_{n-i}\right)\right),
$$

onde:

$$
\alpha_{n-i}=\frac{e^{(i+1) A \Delta t}}{\Delta t} \int_{0}^{\Delta t} \prod_{\substack{k=1 \\ k \neq i}}^{r-2} \frac{\tau+k \Delta t}{(k-i) \Delta t} d \tau \quad ; \quad-1 \leq i \leq r-2
$$

Assim, utilizando o método de Lagrange de segunda ordem, ou seja, $r=3$, o que resulta em $\alpha_{n+1}=\frac{1}{2}$ e $\alpha_{n}=\frac{e^{A \Delta t}}{2}, \log \mathrm{O}:$

$$
\mathbf{y}_{n+1}=\underbrace{e^{A \Delta t}\left(\mathbf{y}_{n}+\frac{\Delta t}{2} \mathbf{S}\left(\mathbf{y}_{n}\right)\right)}_{\text {Termo Explicito }}+\underbrace{\frac{\Delta t}{2} \mathbf{S}\left(\mathbf{y}_{n+1}\right)}_{\text {Termo Implicito }}
$$

Para resolver a exponencial da matriz utiliza-se, neste trabalho, a aproximação pela decomposição espectral da matriz $\boldsymbol{A}$, uma vez que a matriz $\boldsymbol{A}$ é diagonalizável. Então, existe uma matriz $\boldsymbol{P}$ dos autovetores inversível, tal que:

$$
\boldsymbol{A}=\boldsymbol{P} \boldsymbol{\Lambda} \boldsymbol{P}^{-1},
$$

onde $\boldsymbol{\Lambda}$ é uma matriz diagonal dos autovalores da matriz $\boldsymbol{A}, \boldsymbol{P}$ é a matriz associada dos autovetores e $\boldsymbol{P}^{-1}$ é a matriz inversa dos autovetores da matriz $\boldsymbol{A}$, respectivamente. Logo, em vez de determinar $e^{A \Delta t}$, avalia-se $\boldsymbol{P} e^{\Lambda \Delta t} \boldsymbol{P}^{-1}$. 
Aqui cabe uma observação da solução encontrada na equação (21). Ela está na forma implícita o que garante uma maior estabilidade para superar a rigidez do problema proposto. Além disso, a solução é genérica para qualquer tipo de fonte, inclusive não lineares.

\section{RESULTADOS E DISCUSSÃO}

Nesta seção, apresentam-se os resultados numéricos para a metodologia proposta e comparam-se com resultados existentes na literatura. São obtidos os resultados para seis grupos de precursores de nêutrons atrasados para as reatividades do tipo: rampa, zig-zag, senoidal, fonte pulsada e, por fim, testa-se uma fonte externa para a reatividade do tipo rampa negativa. Para cada caso de reatividade estudado é apresentada uma tabela com os resultados obtidos para a densidade de nêutrons e para o caso da fonte externa os resultados são apresentados na forma de gráfico.

Para todos os casos testes foram utilizados os parâmetros cinéticos estudados na obra Tumelero [17]. Considera-se a densidade de nêutrons inicial com $n_{0}=1 \mathrm{~cm}^{3}$. O software utilizado para implementação do algoritmo foi o Scilab 6.0.1 juntamente com um computador que possui essas configurações: Intel(R) Core(TM) i5-7200U 2.5GHz, 8GB de RAM, Sistema operacional de 64 bits e processador com base em x64.

Essas diferentes reatividades podem simular a retirada e inserção de barras de controle, afim de controlar a reação em cadeia, possibilitando um aumento ou diminuição da potência do reator. Para o caso com fonte externa, simula-se uma possível configuração subcrítica, ao qual necessita-se de uma fonte externa para manter-se a reação em cadeia.

\subsection{Inserção de Reatividade Rampa}

Para o primeiro caso teste considera-se uma inserção de reatividade linear $\rho(t)=a t$, onde $a=0,1 \beta$. Usa-se como passo de tempo $\Delta t=10^{-7} \mathrm{~s}$. Comparam-se os resultados em $\mathrm{cm}^{-3}$ com os métodos PAM, com o passo de tempo $\Delta t=0,0001 \mathrm{~s}$, e o BEFD mostrados na tabela 1 até $11 \mathrm{~s}$. 
Tabela 1: Densidade de nêutrons em $\mathrm{cm}^{-3}$ para inserção de reatividade rampa com $\rho=0,1 \beta t$.

\begin{tabular}{cccc}
\hline & FII & PAM & BEFD \\
$\mathrm{t}(\mathrm{s})$ & $\Delta t=0,0000001 s$ & $\Delta t=0,0001 s$ & \\
\hline 2 & $\mathbf{1 , ~ 3 3 8 2 0 0 1 7 8}$ & 1,33820005 & 1,338200050 \\
4 & $\mathbf{2 , ~ 2 2 8 4 4 1 5 2 7}$ & 2,228441895 & 2,228441897 \\
6 & $\mathbf{5 , 5 8 2 0 5 0 6 8 3}$ & 5,582052438 & 5,582052449 \\
8 & $\mathbf{4 2 , \mathbf { 7 8 6 2 6 0 4 5 }}$ & 42,78629544 & 42,78629573 \\
10 & $\mathbf{4 5 1 1 6 6 , \mathbf { 4 0 7 4 }}$ & 451164,0975 & 451163,6239 \\
11 & $\mathbf{1 , 7 9 2 2 4 8 3 6 1 E + 1 6}$ & $1,792327126 \mathrm{E}+16$ & $1,792213607 \mathrm{E}+16$ \\
\hline
\end{tabular}

De acordo com a tabela percebe-se que o método FII aproxima-se bem dos resultados quando comparado com o método PAM [17] e o BEFD [4], concordando de quatro a seis casas decimais, sendo os melhores resultados no tempo $2 s$ e $4 s$, concordando seis casas decimais, o que pode-se considerar um ótimo resultado em física de reatores.

\subsection{Inserção de Reatividade Zig-Zag}

No segundo caso, resolvem-se as ECPN com reatividade do tipo zig-zag, dada por:

$$
\rho(t)= \begin{cases}0,0075 t, & 0 \leq t \leq 0,5, \\ -0,0075(t-0,5)+0,00375, & 0,5 \leq t \leq 1, \\ 0,0075(t-1), & 1 \leq t \leq 1,5 \\ 0,00375, & t \geq 1,5 t\end{cases}
$$

Utiliza-se o passo de tempo $\Delta t=0,0000001 \mathrm{~s}$ e comparam-se com os métodos PAM, com o passo de tempo $\Delta t=0,0001 s$, e o Enhanced Piecewise Constante Approximation (EPCA) apresentado em [15]. Os resultados são apresentados para a densidade de nêutrons em até $2 s$ na tabela 2.

Tabela 2: Densidade de nêutrons em $\mathrm{cm}^{-3}$ para inserção de reatividade zig-zag.

\begin{tabular}{llll}
$\mathrm{t}(\mathrm{s})$ & FII & PAM & EPCA \\
& $\Delta \mathrm{t}=10^{-7} \mathrm{~s}$ & $\Delta t=0,0001 s$ & 1,721422422 \\
\hline 0,5 & $\mathbf{1 , 7 2 1 4 2 2 2 8 1 6}$ & 1,721422393 & 1,211127415 \\
1 & $\mathbf{1 , 2 1 1 1 2 7 3 4 4 1}$ & 1,211127399 & 1,892226140 \\
1,5 & $\mathbf{1 , 8 9 2 2 2 5 9 8 8 2}$ & 1,892226104 & 2,521600530 \\
2 & $\mathbf{2 , 5 2 1 6 0 0 4 9 2 7}$ & 2,521600526 & 2,0 \\
\hline
\end{tabular}


Analisando a tabela deste caso, pode-se concluir que os resultados encontrados assemelham-se com os métodos PAM e BEFD, concordando de cinco a seis casas decimais, sendo no tempo 1,5s o maior erro concordando em cinco casas, o que pode-se considerar um ótimo resultado em física de reatores.

\subsection{Inserção de Reatividade Senoidal}

Para o terceiro caso, considera-se a seis grupos de precursores de nêutrons atrasados e uma inserção periódica de reatividade $\rho(t)=\rho_{0} \operatorname{sen}(t)$, onde $\rho_{0}=0,00073$. A densidade de nêutrons em $\mathrm{cm}^{-3}$ até $10 \mathrm{~s}$, com o passo de tempo $\Delta t=0,0000001 \mathrm{~s}$. Os resultados são apresentados na tabela $3 \mathrm{e}$ comparados com os métodos PAM, com o passo de tempo $\Delta t=0,0001 \mathrm{~s}$, e o Método de Decomposição presente em [13].

Tabela 3: Densidade de nêutrons em $\mathrm{cm}^{-3}$ para inserção de reatividade senoidal com seis grupos de precursores de nêutrons atrasados.

\begin{tabular}{lcccc} 
Reatividade & $\mathrm{t}(\mathrm{s})$ & $\mathbf{F I I}$ & PAM & Decomposição \\
& 1 & $\mathbf{1 , 1 2 3 9 4 0 5 1 3}$ & 1,123940509 & 1,12394 \\
2 & $\mathbf{1 , 1 6 8 8 8 9 5 9 1}$ & 1,168889590 & 1,16884 \\
& 3 & $\mathbf{1 , 0 7 4 4 8 4 6 9 7}$ & 1,074484703 & 1,07442 \\
$\rho$ & $\mathbf{0 , 9 5 3 8 2 9 2 8 6}$ & 0,953829290 & 0,95380 \\
$0,0073 \operatorname{sen}(t)$ & 5 & $\mathbf{0 . 9 0 7 3 5 3 4 9 1 3}$ & 0,907353490 & 0,90737 \\
& 6 & $\mathbf{0 , 9 6 1 5 3 9 5 7 2 4}$ & 0,961539576 & 0,96158 \\
& 7 & $\mathbf{1 , 0 8 7 4 5 8 9 0 5 1}$ & 1,087458911 & 1,08749 \\
& 8 & $\mathbf{1 , 1 7 1 6 7 1 2 7 2 9}$ & 1,171671274 & 1,17164 \\
& 9 & $\mathbf{1 , 1 1 1 3 0 4 4 4 2 8}$ & 1,111304437 & 1,11124 \\
& 10 & $\mathbf{0 , 9 8 4 6 8 0 3 1 8 6}$ & 0,984680323 & 0,98464 \\
\hline
\end{tabular}


Ao observar a tabela deste caso, pode-se notar que os resultados encontrados aproximam-se dos métodos PAM e Decomposição, concordando de seis a oito casas decimais com o método PAM, sendo no tempo $2 s$ o menor erro, concordando em oito casas e no tempo $3 s$ o maior erro, concordando em seis casas, o que pode ser considerado um ótimo resultado em física de reatores. Em comparação com o método de Decomposição, concorda de três a cinco casas decimais, sendo no tempo $1 s$ o menor erro, concordando em todas as casas e, no tempo $9 s$, o maior erro, concordando três casas, o que pode ser considerado um bom resultado em física de reatores.

\subsection{Inserção de Reatividade Fonte Pulsada}

Para o quarto caso de reatividade do tipo fonte pulsada, considera-se um grupo de precursores de nêutrons atrasados em um reator térmico com $\beta=0,006502, \lambda=0,077 / s, \Lambda=5.10^{-4} s$ e reatividade dada por :

$$
\rho(t)=\left\{\begin{aligned}
4 \beta \exp \left(-2 t^{2}\right), & t<1 s \\
0, & t>1 s
\end{aligned}\right\}
$$

Utiliza-se para o FII o passo de tempo $\Delta t=0,0000001 s$. A tabela 4 mostra os resultados obtidos para a densidade de nêutrons em $\mathrm{cm}^{-3}$ até $3 \mathrm{~s}$ comparados com os métodos PAM, com o passo de tempo $\Delta t=0,0001 s$, e o BEFD.

Tabela 4: Densidade de nêutrons em $\mathrm{cm}^{-3}$ para inserção de reatividade fonte pulsada.

\begin{tabular}{cccc}
\hline \multirow{2}{*}{$(\mathrm{s})$} & FII & PAM & BEFD \\
& $\Delta \mathrm{t}=10^{-7} \mathrm{~s}$ & $\Delta t=0,0001 s$ & \\
\hline 0,5 & $\mathbf{9 3 8 0 0 5 3 , \mathbf { 8 4 2 }}$ & 9380592,423 & 9380044,272 \\
0,8 & $\mathbf{1 6 9 4 7 7 9 6 0 , 3}$ & 169487791,0 & 169477616,1 \\
1 & $\mathbf{1 0 7 5 1 3 3 5 1 , \mathbf { 0 }}$ & 107519618,5 & 107513170,4 \\
2 & $\mathbf{4 8 3 4 1 1 6 , 0 2 4}$ & 4834396,081 & 4834106,369 \\
3 & $\mathbf{4 8 3 3 9 0 1 , 9 9 2}$ & 4834182,039 & 4833892,339 \\
\hline
\end{tabular}


Examinando a tabela percebe-se que o método FII aproxima-se bem dos resultados dos métodos PAM e BEFD, concordando de três a cinco dígitos com o método PAM, sendo em $1 s$ o menor erro, concordando cinco dígitos e em $3 s$ o maior erro, concordando três dígitos. Em comparação com o BEFD tem-se que o método FII concorda de quatro a seis casas decimais, sendo em $0,8 s$ o menor erro, concordando seis casas e em $3 s$ o maior erro, quatro casas, que pode ser considerado em ambas comparações um bom resultado em física de reatores.

\subsection{Inserção de Reatividade tipo Rampa negativa com Fonte Externa}

Para o quinto caso considera-se a inserção de uma reatividade do tipo rampa negativa dada por $\rho(t)=a t$, sendo $a=-0,001 \beta$, e uma fonte externa constante $S=30 \mathrm{~cm}^{-3}$ ligada aos $4 \mathrm{~s}$. Os resultados foram calculados até o tempo de 100s e são apresentados na Figura 2.

Pode-se observar um salto na densidade logo após o ligamento da fonte, atingindo o máximo em aproximadamente $1,28 \mathrm{~cm}^{-3}$ no tempo de $40 \mathrm{~s}$, seguido de um decréscimo lento a partir desse ponto. Comportamento esse esperado devido a inserção negativa de reatividade do tipo rampa negativa. 


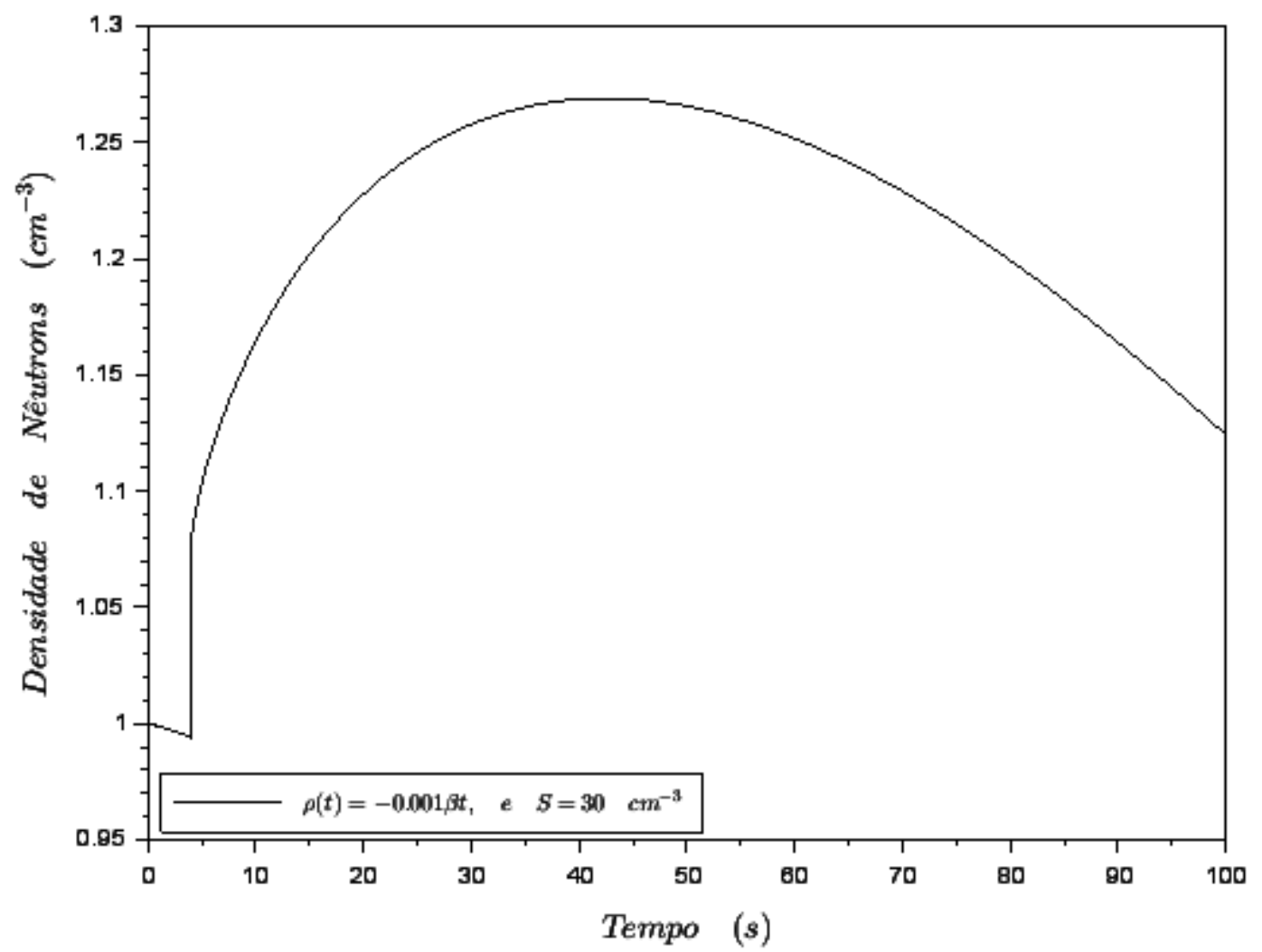

Figura 2 : Densidade de nêutrons para $(\rho(t)=-0,001 \beta t)$ e uma fonte externa constante ligada $S=30 \mathrm{~cm}^{-3}$ em 4 segundos

\section{CONCLUSÕES}

Neste trabalho as ECPN foram resolvidas com sucesso pelo Método de Fator de Integração Implícito, considerando 6 grupos de precursores de nêutrons atrasados, reatividade constante e variável com o tempo com adição de um termo fonte. O método se tornou robusto e eficiente para o cálculo da densidade para diferentes reatividades e tempos, considerando interpolação por Lagrange de baixa ordem para gerar resultados com boa concordância com resultados apresentados na literatura. A rigidez foi superada com certa facilidade, não só pela formulação implícita do método, 
mas pelo auxílio da continuação analítica. Além disso, a dependência temporal na reatividade foi tratada com a aproximação constante por partes, seguindo a ideia clássica do método PCA.

Os resultados apresentados sem fonte externa serviram para mostrar a viabilidade do método para posterior simulação e testes com fontes. Nesse sentido, o método apresentado neste trabalho torna-se viável para resolver as Equações da Cinética Pontual de Nêutrons, incluindo termos fontes. Mesmo não simulando casos não lineares, a metodologia é geral, podendo incluir não linearidades.

Cabe ressaltar que existe muita pouca referência na literatura para resolver as ECPN, incluindo termos fontes. Os autores estão cientes que essas fontes precisam ser melhor investigadas para modelar de forma mais realística esses sistemas subcríticos acionados por fontes externas. Entretanto, o método proposto se torna viável para simulações futuras, inclusive considerando fontes não lineares.

Portanto, os autores pretendem expandir essa metodologia para modelos mais realísticos acionados por fontes externas que incluam: feedback de temperatura e venenos absorvedores de nêutrons, a fim de mostrar a viabilidade desses novos reatores regeneradores de rejeitos radioativos. 


\section{AGRADECIMENTOS}

Os autores agradecem à FAPERGS e à Universidade Federal de Pelotas pelo apoio financeiro e suporte, em especial ao Programa de Pós-Graduação em Modelagem Matemática da referida universidade.

\section{REFERÊNCIAS}

[1] ABOANBER, A. E.; HAMADA, Y. M. Power series solution (PWS) of nuclear reactor dynamics with newtonian temperature feedback. Annals of Nuclear Energy, p. 1111-1122, 2003.

[2] ABOANBER, A. E.; HAMADA, Y. M. On Pade' Approximations to the Exponential Function and Application to the Point Kinetics Equations. Progress in Nuclear Energy, v. 44, No. 4, p. 347-368, 2004.

[3] CHAO, Y.; ATTARDT, A. A resolution of the stiffness problem of reactor kinetics. Nuclear Science and Engineering. p. 40-46, 2017.

[4] DUDERSTADT J. J.; HAMILTON, L. J. Nuclear Reactor Analysis, John Wiley \& Sons, New York, 1976.

[5] GANAPOL, B. D. The refined way to solve the reactor point kinetics equations for imposed reactivity insertions. Nuclear Technology and Radiation Protection, v. 24, p. 157-166, 2009.

[6] GANAPOL, B. D.; PICCA, P. A highly accurate benchmark for reactor point kinetics with feedback. In: 2010. Annals of Nuclear Energy, The 17th Pacific Basin Nuclear Conference: 17th PBNC: Cancun: México, 2010.

[7] GANAPOL, B. D. A highly accurate algorithm for the solution of the point kinetics equations. Annals of Nuclear Energy, v.62, p.564-571, 2013.

[8] SÁNCHEZ, J. On the numerical solution of the point reactor kinetics equations by generalized Runge-Kutta methods. Nuclear Science and Engineering, v. 103, p. 94-99, 1989.

[9] QUINTERO-LEYVA, B. CORE: a numerical algorithm to solve the point kinetics equations. Annals of Nuclear Energy, v. 35, p. 2136-2138, 2008. 
[10] NAHLA, A. A. Generalization of the analytical exponential model to solve the point kinetics equations of Be- and D2O-moderated reactors. Nuclear Engineering and Design, v. 238, p. 2648-2653, 2008.

[11] LI, H.; CHEN, W.; LUO, L.; ZHU, Q. A new integral method for solving the point reactor neutron kinetics equations. Annals of Nuclear Energy, v. 36, p. 427-432, 2009.

[12] NAHLA, A. A. An efficient technique for the point reactor kinetics equations with Newtonian temperature feedback effects. Annals of Nuclear Energy, v. 38, p. 2810-2817, 2011.

[13] PETERSEN, C. Z.; DULLA, S.; VILHENA, M. T. B.; RAVETTO, P. An analytical solution of the point kinetics equations with time-variable reactivity by the decomposition method. Progress in Nuclear Energy, v. 53, p. 1091-1094, 2011.

[14] KINARD, M.; ALLEN, E. Efficient numerical solution of the point kinetics equations in nuclear reactor dynamics. Annals of Nuclear Energy, v. 31, p. 1039-1051, 2004.

[15] PICCA, P.; FURFARO, R.; GANAPOL, B. A highly accurate technique for the solution of the non-linear point kinetics equations. Annals of Nuclear Energy, v. 58, p. 43-53, 2013.

[16] SILVA, J. J. A.; ALVIM, A. C. M.; VILHENA, M. T. M. B.; BODMANN, B. E. J.; PETERSEN, C. Z. On a closed-form solution of the point kinetics equations with reactivity feedback of temperature. International Journal of Nuclear Energy, Science and Technology (Print), v. 8, p. 131-145, 2014.

[17] TUMELERO, F.; PETERSEN, C. Z.; GONÇALVES, G. A. and SCHRAMM, M. Polynomial approach method to solve the neutron point kinetics equations with use of the analytic continuation. KERNTECHNIK, v. 81, p. 662-670, 2016.

[18] XOUBI, N. Neutronic design study of accelerator driven system (ADS) for Jordan subcritical reactor as a neutron source for nuclear research. Applied Radiation and Isotopes, v. 131, p. 71 $76,2018$.

[19] AL QAAOD, A. A.; GULIK, V. ${ }^{226} \mathrm{Ra}$ irradiation to produce ${ }^{225} \mathrm{Ac}$ and ${ }^{213} \mathrm{Bi}$ in an acceleratordriven system reactor. Nuclear Science and Techniques, v. 31, 44, 2020. 